\title{
OPTIMIZATION OF THE SCANNING PROTOCOL IN MUSCULOSKELETAL ULTRASOUND
}

\author{
Standardization of the acquisition parameters
}

\author{
Dott. Alessandroni Maddalena, Dott. Collocola Alessio, Dott. Durante Stefano
}

KEYWORDS: Ultrasound, Optimization, Skeletal Muscle, Dynamic Range

\section{ABSTRACT}

The main limit of the ultrasound resides on the fact that it is a strictly operator-dependent method, and, against the growing discrepancy in the execution methods, the application of a specific protocol for the anatomical area of interest has the function of implementing the achievement of the diagnostic result.

This experimentation aims to optimize the parameters of the ultrasound equipment, remaining placed in a strictly technical context, without trying to emulate in any way the diagnostic ability of the medical professional.

The first step was mainly focused on ultrasound training and in particular on acquiring a good mastership of the technical image parameters.

Subsequently there were performed some scans on five volunteer patients in various anatomical areas according to two different orientations of the ultrasound transducer. The images were acquired at first by the use of the presets already placed on the ultrasound equipment since the installation.

The next step was to create a new ultrasound protocol for musculoskeletal tissue changing the values of the technical parameters of the image by scanning the five patients previously recruited.

The final step concerned the comparison with the medical professional who was required to scan the same five patients in the same anatomical sections and according to the same scan plans previously set.

This experimentation compares the three protocols above mentioned, pursuing the aim to set up an execution protocol for the ultrasound study of musculoskeletal tissue by defining the right setting for the main technical image parameters in order to optimize the performance of the exam, to reduce the time required to complete the survey and to obtain good quality images.

\section{INTRODUCTION}

Ultrasound is a diagnostic imaging technique that exploits the physical properties and biological interaction of ultrasound to generate an image. Ultrasounds are not radiations but mechanical vibrations, longitudinal elastic waves of rarefaction and compression that propagate with variable speed according to the density and the acoustic impedance of the tissue. The widespread diffusion of the ultrasound scanning, the low cost, the biological non-invasiveness and the simplicity of using this technology are just some of the factors that have determined an uncontrolled growth in the requests for ultrasounds investigations for a diagnostic purpose. And it is not difficult to imagine how many are the performances guaranteed to patients, if compared to the number of Ultrasound equipments actually used. The current Ultrasound equipments have application setting systems (preset) that have the function of personalizing some parameters so they can be adapted in the best way to the use by the healthcare professional. Usually the manufacturer has the task to install the various presets in order to optimize the performance of the equipment and to speed up the execution of the diagnostic examination. However, the presets installed on the equipment at the time of purchase do not always meet the needs of the operator and require to be updated and revisited before being able to use them.

Regarding the quality of the images, the Ultrasound post processing does not allow the further processing of the images as in the other diagnostic methods, reducing the post production to a few functions that can be carried out on the numeric values stored in the image matrix; exactly for this reason it's essential to acquire the images in an optimal way already in the pre-processing phase, attributing the right values to the technical image parameters according to the districts investigated.

Moreover it can be added what is the main limitation of ultrasound: the close operator-equipement dependence; in order that the ultrasound information can be correctly interpreted, in fact, special abilities of manual skills, observation skills, image culture and clinical experience are required. This study aims to establish an applicable scan protocol to the study of musculoskeletal tissue, optimizing the fundamental parameters for good image quality.

\section{MATERIALS AND METHODS}

This experimental study was carried out mainly at the Rizzoli Orthopedic Institute in Bologna in the period between May and October 2019.

Five volunteer patients between the ages of 20 and 30 were recruited. It is important to remember the total absence of side effects due to the use of ultrasounds for diagnostic investigations, so this experiment can be actually considered without any kind of consequences for the patients recruited. For each patient there were scanned overall:

- left gluteus;

- $\quad$ left popliteal fossa;

- $\quad$ left femoral quadriceps.

These are anatomical districts in which the ultrasound undoubtedly represents the examination of first instance thanks also to the superficiality of the structures, which makes it possible to use high frequency ultrasounds. The choice of these sections 


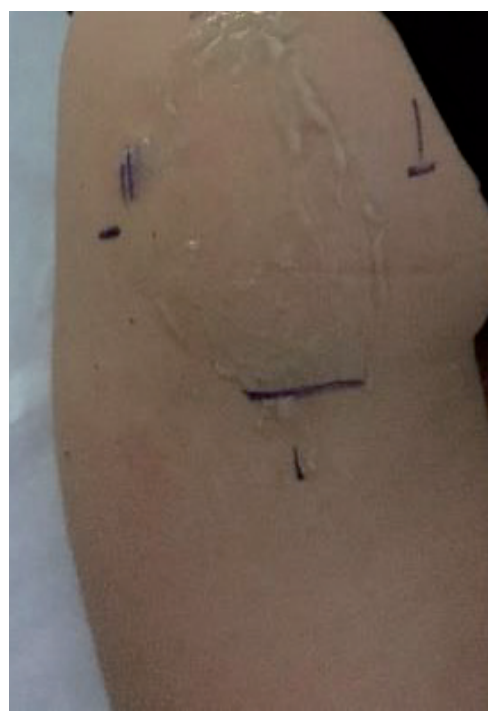

Fig. 1 - Left gluteus.

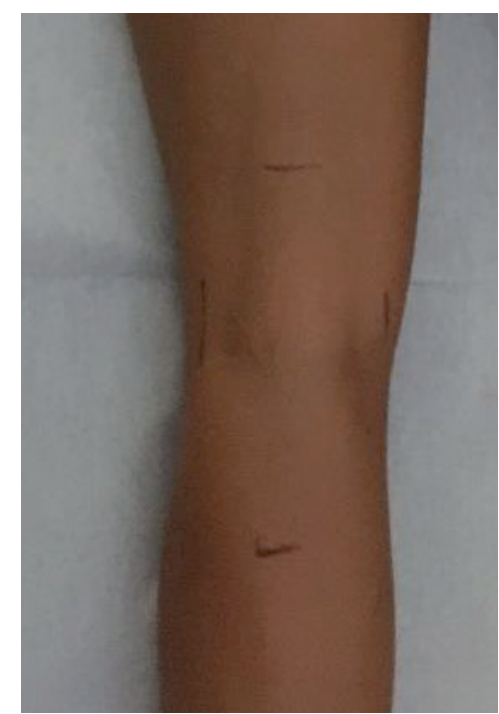

Fig. 2 - Left popliteal fossa.

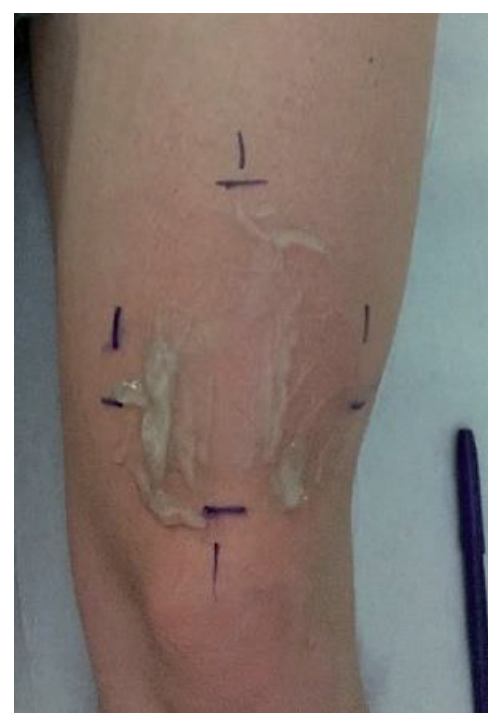

Fig. 3 - Left femoral quadriceps. has allowed us to study areas with different thicknesses and, consequently, to be able to obtain final values starting from the study of anatomical areas that are as different as possible.

The scans were performed using the Philips Affiniti 50 ultrasound system, an ergonomic system that is easy to use and widely used as it is strong enough to withstand the daily stresses due to the high volume of patients. A multifrequency linear transducer $(5-12 \mathrm{MHz})$ was used, ideal for the study of pathologies affecting the musculoskeletal system, and the scans were carried out along the sagittal and transverse orientation (long axis and short axis).

Together with an ultrasound Product Specialist, all the parameters that describe an ultrasound image

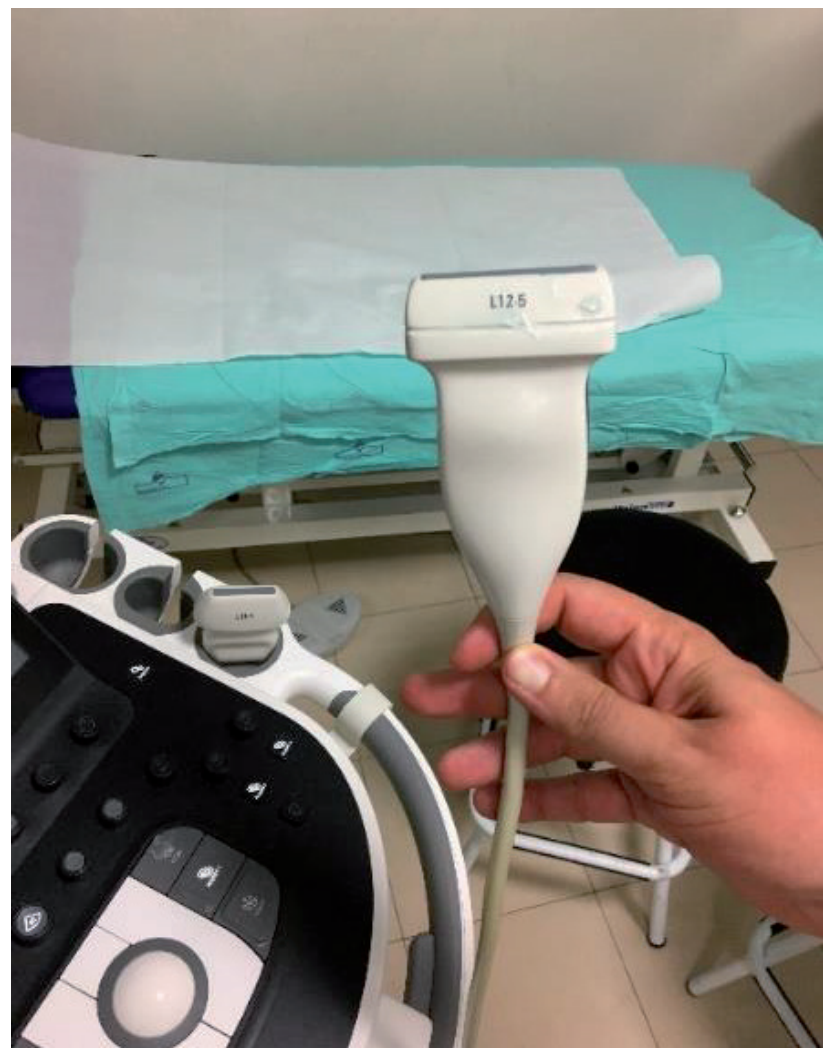

Fig. 4 - Philips Affiniti 50 Ultrasound System. were analyzed and subsequently were considered for this study the technical image parameters that most influence the iconographic result:

- Gain, which is measured in \% and allows to adjust the intensity with which the ultrasound system perceives the return echo signals.

- The Depth, measured in $\mathrm{cm}$, which determines how much distance from the transducer inside the body is displayed on the monitor. It allows to focus on the part to analyze, making sure that the most superficial structures are enlarged and the resolution is increased.

- The Focus, also measured in $\mathrm{cm}$, is responsible for the optimization of the lateral resolution at a certain depth. The best spatial resolution of

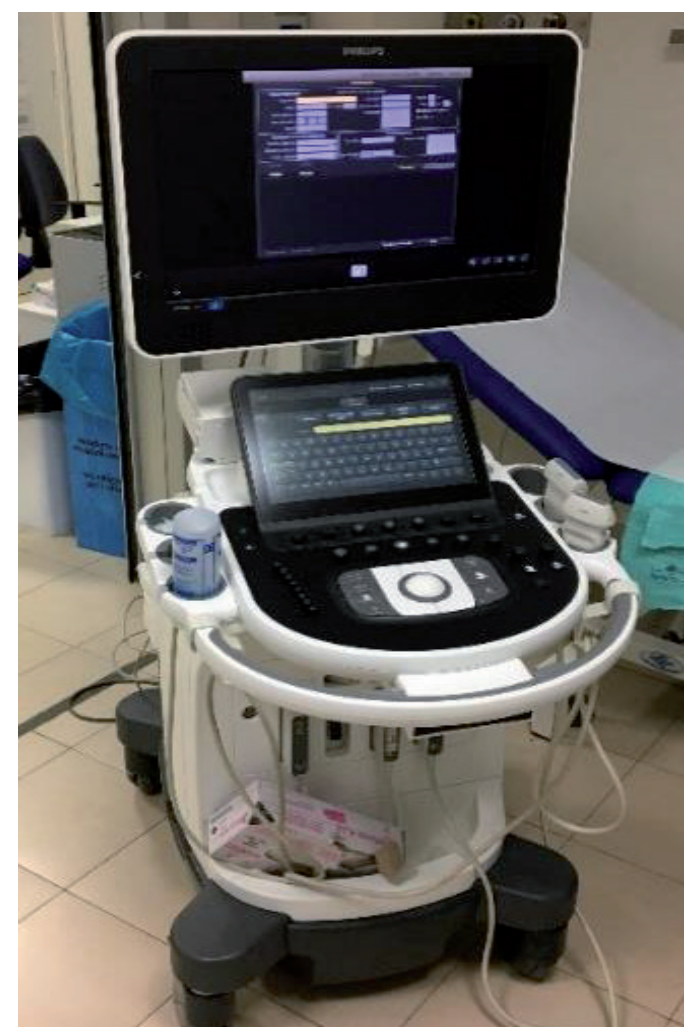

Fig. 5 - Linear transducer (12-5 MHz) ideal for studying the musculoskeletal system. 
Transducer 5-12 MHz

Preset on the US Equipment

\begin{tabular}{|c|c|}
\hline Gain & $46 \%$ \\
\hline Depth & $3 \mathrm{~cm}$ \\
\hline Focus & $1,5 \mathrm{~cm}$ \\
\hline Dynamic Range & $56 \mathrm{~dB}$ \\
\hline
\end{tabular}

Tab. 1 - Property of the preset installed on the ultrasound equipment concerning the study of musculoskeletal tissue.

an object is reached in the focal area, where the ultrasound beam is maximally narrow. Generally, the focus is already preset for a certain use, but it may be necessary to adjust it according to the structures being examined.

- The Dynamic Range, measured in decibell, which refers to the range of echo signals arriving on the screen. Increasing the dynamic range reduces the contrast resolution, while decreasing it will increase the contrast resolution.

Three different series of scans were carried out; the first is the one that involves the use of the preset installed on the ultrasound equipment at the time of purchase, the second involves the application of the experimental preset, and finally the third that involves the figure of the medical professional. Each series of scans involves the acquisition of each single anatomical district along the sagittal and transverse scan plans.

The preset dedicated to the study of the structures of the musculoskeletal system originally installed on the ultrasound equipement had the following characteristics:

The scans performed by using the preset installed on the ultrasound equipement were then followed by scans performed with different settings of gain, depth, focus and dynamic range values.

Several attempts have been made on each patient to establish the values of the technical parameters that define in the best way the good image quality. Following this scans, the average of the values obtained for each single parameter was calculated until a final protocol was drawn up:

The next step of the experimentation sees the involvement of the figure of the medical professional, who was asked to scan the same anatomical sections of the five patients already recruited in the previous two studies.

No particular indications were reported to the operator to make sure that the scans were performed in complete autonomy, following the habits related to the

\begin{tabular}{|c|c|c|c|c|c|c|}
\cline { 2 - 7 } \multicolumn{1}{c|}{} & \multicolumn{2}{c|}{ Gluteus } & \multicolumn{2}{c|}{ Popliteal Fossa } & \multicolumn{2}{c|}{ Femoral Quadriceps } \\
\hline Patient 1 & sagittal & transverse & sagittal & transverse & sagittal & transverse \\
\hline Gain & $45 \%$ & $46 \%$ & $48 \%$ & $45 \%$ & $48 \%$ & $46 \%$ \\
\hline Depth & $5 \mathrm{~cm}$ & $6 \mathrm{~cm}$ & $4,5 \mathrm{~cm}$ & $3,5 \mathrm{~cm}$ & $3,5 \mathrm{~cm}$ & $4 \mathrm{~cm}$ \\
\hline Focus & $3 \mathrm{~cm}$ & $3,5 \mathrm{~cm}$ & $2,25 \mathrm{~cm}$ & $2 \mathrm{~cm}$ & $1,75 \mathrm{~cm}$ & $2 \mathrm{~cm}$ \\
\hline $\begin{array}{c}\text { Dynamic } \\
\text { Range }\end{array}$ & $50 \mathrm{~dB}$ & $50 \mathrm{~dB}$ & $50 \mathrm{~dB}$ & $50 \mathrm{~dB}$ & $50 \mathrm{~dB}$ & $50 \mathrm{~dB}$ \\
\hline
\end{tabular}

Tab. 2 - Collected data relating to scans with optimized preset on Patient 1.

\begin{tabular}{|c|c|c|c|c|c|c|}
\cline { 2 - 7 } \multicolumn{1}{c|}{} & \multicolumn{2}{c|}{ Gluteus } & \multicolumn{2}{c|}{ Popliteal Fossa } & \multicolumn{2}{c|}{ Femoral Quadriceps } \\
\hline Patient 2 & sagittal & transverse & sagittal & transverse & sagittal & transverse \\
\hline Gain & $54 \%$ & $55 \%$ & $52 \%$ & $51 \%$ & $48 \%$ & $45 \%$ \\
\hline Depth & $4 \mathrm{~cm}$ & $6 \mathrm{~cm}$ & $4,5 \mathrm{~cm}$ & $5 \mathrm{~cm}$ & $4,5 \mathrm{~cm}$ & $3,5 \mathrm{~cm}$ \\
\hline Focus & $2,5 \mathrm{~cm}$ & $3,5 \mathrm{~cm}$ & $2 \mathrm{~cm}$ & $1,5 \mathrm{~cm}$ & $2,15 \mathrm{~cm}$ & $2 \mathrm{~cm}$ \\
\hline $\begin{array}{c}\text { Dynamic } \\
\text { Range }\end{array}$ & $45 \mathrm{~dB}$ & $45 \mathrm{~dB}$ & $48 \mathrm{~dB}$ & $47 \mathrm{~dB}$ & $46 \mathrm{~dB}$ & $44 \mathrm{~dB}$ \\
\hline
\end{tabular}

Tab. 3 - Collected data relating to scans with optimized preset on Patient 2.

\begin{tabular}{|c|c|c|c|c|c|c|}
\cline { 2 - 7 } \multicolumn{1}{c|}{} & \multicolumn{2}{c|}{ Gluteus } & \multicolumn{2}{c|}{ Popliteal Fossa } & \multicolumn{2}{c|}{ Femoral Quadriceps } \\
\hline Patient 3 & sagittal & transverse & sagittal & transverse & sagittal & transverse \\
\hline Gain & $51 \%$ & $53 \%$ & $52 \%$ & $52 \%$ & $52 \%$ & $48 \%$ \\
\hline Depth & $4 \mathrm{~cm}$ & $4,5 \mathrm{~cm}$ & $4 \mathrm{~cm}$ & $4 \mathrm{~cm}$ & $3,5 \mathrm{~cm}$ & $3,5 \mathrm{~cm}$ \\
\hline Focus & $2,25 \mathrm{~cm}$ & $2,25 \mathrm{~cm}$ & $2 \mathrm{~cm}$ & $2 \mathrm{~cm}$ & $2,25 \mathrm{~cm}$ & $1,75 \mathrm{~cm}$ \\
\hline $\begin{array}{c}\text { Dynamic } \\
\text { Range }\end{array}$ & $44 \mathrm{~dB}$ & $44 \mathrm{~dB}$ & $49 \mathrm{~dB}$ & $49 \mathrm{~dB}$ & $44 \mathrm{~dB}$ & $44 \mathrm{~dB}$ \\
\hline
\end{tabular}

Tab. 4 - Collected data relating to scans with optimized preset on Patient 3.

\begin{tabular}{|c|c|c|c|c|c|c|}
\cline { 2 - 7 } \multicolumn{1}{c|}{} & \multicolumn{2}{c|}{ Gluteus } & \multicolumn{2}{c|}{ Popliteal Fossa } & \multicolumn{2}{c|}{ Femoral Quadriceps } \\
\hline Patient 4 & sagittal & transverse & sagittal & transverse & sagittal & transverse \\
\hline Gain & $55 \%$ & $56 \%$ & $49 \%$ & $53 \%$ & $52 \%$ & $54 \%$ \\
\hline Depth & $5 \mathrm{~cm}$ & $6 \mathrm{~cm}$ & $4 \mathrm{~cm}$ & $5 \mathrm{~cm}$ & $4 \mathrm{~cm}$ & $4,5 \mathrm{~cm}$ \\
\hline Focus & $2,75 \mathrm{~cm}$ & $3,25 \mathrm{~cm}$ & $2,5 \mathrm{~cm}$ & $3 \mathrm{~cm}$ & $2,25 \mathrm{~cm}$ & $2,75 \mathrm{~cm}$ \\
\hline $\begin{array}{c}\text { Dynamic } \\
\text { Range }\end{array}$ & $48 \mathrm{~dB}$ & $48 \mathrm{~dB}$ & $60 \mathrm{~dB}$ & $45 \mathrm{~dB}$ & $48 \mathrm{~dB}$ & $39 \mathrm{~dB}$ \\
\hline
\end{tabular}

Tab. 5 - Collected data relating to scans with optimized preset on Patient 4. 


\begin{tabular}{|c|c|c|c|c|c|c|}
\cline { 2 - 7 } \multicolumn{1}{c|}{} & \multicolumn{2}{c|}{ Gluteus } & \multicolumn{2}{c|}{ Popliteal Fossa } & \multicolumn{2}{c|}{ Femoral Quadriceps } \\
\hline Patient 5 & sagittal & transverse & sagittal & transverse & sagittal & transverse \\
\hline Gain & $55 \%$ & $55 \%$ & $48 \%$ & $48 \%$ & $55 \%$ & $54 \%$ \\
\hline Depth & $4,5 \mathrm{~cm}$ & $4,5 \mathrm{~cm}$ & $5 \mathrm{~cm}$ & $5 \mathrm{~cm}$ & $4 \mathrm{~cm}$ & $3,5 \mathrm{~cm}$ \\
\hline Focus & $2,5 \mathrm{~cm}$ & $2,5 \mathrm{~cm}$ & $3 \mathrm{~cm}$ & $3 \mathrm{~cm}$ & $2,25 \mathrm{~cm}$ & $2 \mathrm{~cm}$ \\
\hline $\begin{array}{c}\text { Dynamic } \\
\text { Range }\end{array}$ & $45 \mathrm{~dB}$ & $45 \mathrm{~dB}$ & $45 \mathrm{~dB}$ & $45 \mathrm{~dB}$ & $44 \mathrm{~dB}$ & $46 \mathrm{~dB}$ \\
\hline
\end{tabular}

Tab. 6 - Collected data relating to scans with optimized preset on Patient 5.

\begin{tabular}{|c|c|c|c|c|c|c|}
\cline { 2 - 7 } \multicolumn{1}{c|}{} & \multicolumn{2}{c|}{ Gluteus } & \multicolumn{2}{c|}{ Popliteal Fossa } & \multicolumn{2}{c|}{ Femoral Quadriceps } \\
\hline Average & sagittal & transverse & sagittal & transverse & sagittal & transverse \\
\hline Gain & $52 \%$ & $53 \%$ & $50 \%$ & $50 \%$ & $51 \%$ & $49 \%$ \\
\hline Depth & $4,5 \mathrm{~cm}$ & $5,4 \mathrm{~cm}$ & $4,4 \mathrm{~cm}$ & $4,5 \mathrm{~cm}$ & $3,9 \mathrm{~cm}$ & $3,8 \mathrm{~cm}$ \\
\hline Focus & $2,6 \mathrm{~cm}$ & $3 \mathrm{~cm}$ & $2,35 \mathrm{~cm}$ & $2,3 \mathrm{~cm}$ & $2,13 \mathrm{~cm}$ & $2,1 \mathrm{~cm}$ \\
\hline $\begin{array}{c}\text { Dynamic } \\
\text { Range }\end{array}$ & $46,4 \mathrm{~dB}$ & $46,4 \mathrm{~dB}$ & $50,4 \mathrm{~dB}$ & $47,2 \mathrm{~dB}$ & $46,4 \mathrm{~dB}$ & $44,6 \mathrm{~dB}$ \\
\hline
\end{tabular}

Tab. 7 - Average of the collected data relating to scans with optimized preset.

\begin{tabular}{|c|c|c|c|c|c|c|}
\cline { 2 - 7 } \multicolumn{1}{c|}{} & \multicolumn{2}{c|}{ Gluteus } & \multicolumn{2}{c|}{ Popliteal Fossa } & \multicolumn{2}{c|}{ Femoral Quadriceps } \\
\hline Patient 1 & sagittal & transverse & sagittal & transverse & sagittal & transverse \\
\hline Gain & $45 \%$ & $39 \%$ & $45 \%$ & $47 \%$ & $47 \%$ & $47 \%$ \\
\hline Depth & $5 \mathrm{~cm}$ & $5 \mathrm{~cm}$ & $5 \mathrm{~cm}$ & $5,5 \mathrm{~cm}$ & $5,5 \mathrm{~cm}$ & $5,5 \mathrm{~cm}$ \\
\hline Focus & $2 \mathrm{~cm}$ & $2,5 \mathrm{~cm}$ & $3 \mathrm{~cm}$ & $3,5 \mathrm{~cm}$ & $2 \mathrm{~cm}$ & $2 \mathrm{~cm}$ \\
\hline $\begin{array}{c}\text { Dynamic } \\
\text { Range }\end{array}$ & $55 \mathrm{~dB}$ & $55 \mathrm{~dB}$ & $55 \mathrm{~dB}$ & $55 \mathrm{~dB}$ & $55 \mathrm{~dB}$ & $55 \mathrm{~dB}$ \\
\hline
\end{tabular}

Tab. 8 - Collected data relating to scans performed by a medical professional on Patient 1.

\begin{tabular}{|c|c|c|c|c|c|c|}
\cline { 2 - 7 } \multicolumn{1}{c|}{} & \multicolumn{2}{c|}{ Gluteus } & \multicolumn{2}{c|}{ Popliteal Fossa } & \multicolumn{2}{c|}{ Femoral Quadriceps } \\
\hline Patient 2 & sagittal & transverse & sagittal & transverse & sagittal & transverse \\
\hline Gain & $54 \%$ & $53 \%$ & $53 \%$ & $52 \%$ & $46 \%$ & $41 \%$ \\
\hline Depth & $7 \mathrm{~cm}$ & $7 \mathrm{~cm}$ & $7 \mathrm{~cm}$ & $7 \mathrm{~cm}$ & $4,5 \mathrm{~cm}$ & $4,5 \mathrm{~cm}$ \\
\hline Focus & $4,5 \mathrm{~cm}$ & $4,5 \mathrm{~cm}$ & $4,5 \mathrm{~cm}$ & $3,5 \mathrm{~cm}$ & $2,5 \mathrm{~cm}$ & $2,5 \mathrm{~cm}$ \\
\hline $\begin{array}{c}\text { Dynamic } \\
\text { Range }\end{array}$ & $56 \mathrm{~dB}$ & $56 \mathrm{~dB}$ & $56 \mathrm{~dB}$ & $56 \mathrm{~dB}$ & $56 \mathrm{~dB}$ & $56 \mathrm{~dB}$ \\
\hline
\end{tabular}

Tab.9-Collected data relating to scans performed by a medical professional on Patient 2.

\begin{tabular}{|c|c|c|c|c|c|c|}
\cline { 2 - 7 } \multicolumn{1}{c|}{} & \multicolumn{2}{c|}{ Gluteus } & \multicolumn{2}{c|}{ Popliteal Fossa } & \multicolumn{2}{c|}{ Femoral Quadriceps } \\
\hline Patient 3 & sagittal & transverse & sagittal & transverse & sagittal & transverse \\
\hline Gain & $54 \%$ & $54 \%$ & $53 \%$ & $52 \%$ & $56 \%$ & $55 \%$ \\
\hline Depth & $4,5 \mathrm{~cm}$ & $4 \mathrm{~cm}$ & $4,5 \mathrm{~cm}$ & $5 \mathrm{~cm}$ & $5 \mathrm{~cm}$ & $5 \mathrm{~cm}$ \\
\hline Focus & $2,5 \mathrm{~cm}$ & $2,25 \mathrm{~cm}$ & $2,75 \mathrm{~cm}$ & $3,25 \mathrm{~cm}$ & $3 \mathrm{~cm}$ & $3 \mathrm{~cm}$ \\
\hline $\begin{array}{c}\text { Dynamic } \\
\text { Range }\end{array}$ & $48 \mathrm{~dB}$ & $43 \mathrm{~dB}$ & $44 \mathrm{~dB}$ & $44 \mathrm{~dB}$ & $45 \mathrm{~dB}$ & $44 \mathrm{~dB}$ \\
\hline
\end{tabular}

Tab. 10 - Collected data relating to scans performed by a medical professional on Patient 3 ultrasound school he belongs. In this step were collected the following data.

\section{RESULTS \\ AND DISCUSSION}

The data collected in the series of scans allow us to compare three different acquisition protocols; comparing them we obtain the following results.

From the graphical representation above it's easily to see the difference in terms of values between the two columns on the left, representing respectively the optimized preset and the medical protocol, compared to the right column that represents the preset on the ultrasound equipement.

Taking as reference the values used by the medical professional, the inadequacy of the preset installed on the ultrasound equipement can be affirmed with certainty, which is also clearly visible in terms of image quality, as demonstrated by the scans performed.

In this context, the optimized preset that has been tested in this study undoubtedly represents a valid acquisition protocol, and its effective quality was subsequently validated by comparing it with the protocol used by the medical professional, producing satisfactory results: exept a small difference, the optimized preset is almost superimposable on the medical one.

The difference in values between the two protocols may be attributable in the first place to the possibility of a different ultrasound school of belonging, and subsequently to a modus operandi taken over time through the exercise with old generation equipment, which need a bigger thrust on the fundamental image parameters. In fact, nowadays the new generation equipment is able to produce high quality images as well with the reduction of the parameter values. That is the reason why the experimental protocol is represented by slightly lower values than those set by the medical professional.

This study wanted to suggest a starting protocol for ultrasound scans in the musculoskeletal field, which will presumably be followed by a modification of the parameters based on the patient's characteristics. It is important to remember that ultrasound was born as a strictly operator-dependent practice, and a fine knowledge of anatomy, a solid experience of the equipment setting and image culture is also fundamental. For this reason it is necessary to adhere to mandatory "standards", remembering that the first foundation of the quality of an exam stays in the preparation and updating of the person who conduces it. 


\begin{tabular}{|c|c|c|c|c|c|c|}
\cline { 2 - 7 } \multicolumn{1}{c|}{} & \multicolumn{2}{c|}{ Gluteus } & \multicolumn{2}{c|}{ Popliteal Fossa } & \multicolumn{2}{c|}{ Femoral Quadriceps } \\
\hline Patient 4 & sagittal & transverse & sagittal & transverse & sagittal & transverse \\
\hline Gain & $54 \%$ & $52 \%$ & $48 \%$ & $49 \%$ & $52 \%$ & $51 \%$ \\
\hline Depth & $5 \mathrm{~cm}$ & $6 \mathrm{~cm}$ & $4,5 \mathrm{~cm}$ & $4,5 \mathrm{~cm}$ & $5 \mathrm{~cm}$ & $4,5 \mathrm{~cm}$ \\
\hline Focus & $2,75 \mathrm{~cm}$ & $3,25 \mathrm{~cm}$ & $2,5 \mathrm{~cm}$ & $2,5 \mathrm{~cm}$ & $3 \mathrm{~cm}$ & $2,5 \mathrm{~cm}$ \\
\hline $\begin{array}{c}\text { Dynamic } \\
\text { Range }\end{array}$ & $43 \mathrm{~dB}$ & $45 \mathrm{~dB}$ & $42 \mathrm{~dB}$ & $45 \mathrm{~dB}$ & $47 \mathrm{~dB}$ & $46 \mathrm{~dB}$ \\
\hline
\end{tabular}

\begin{tabular}{|c|c|c|c|c|c|c|}
\cline { 2 - 7 } \multicolumn{1}{c|}{} & \multicolumn{2}{c|}{ Gluteus } & \multicolumn{2}{c|}{ Popliteal Fossa } & \multicolumn{2}{c|}{ Femoral Quadriceps } \\
\hline Patient 5 & sagittal & transverse & sagittal & transverse & sagittal & transverse \\
\hline Gain & $53 \%$ & $51 \%$ & $49 \%$ & $49 \%$ & $54 \%$ & $52 \%$ \\
\hline Depth & $4 \mathrm{~cm}$ & $4 \mathrm{~cm}$ & $4,5 \mathrm{~cm}$ & $4,5 \mathrm{~cm}$ & $4 \mathrm{~cm}$ & $3,5 \mathrm{~cm}$ \\
\hline Focus & $2,5 \mathrm{~cm}$ & $2,5 \mathrm{~cm}$ & $2,5 \mathrm{~cm}$ & $2,25 \mathrm{~cm}$ & $2,25 \mathrm{~cm}$ & $2,5 \mathrm{~cm}$ \\
\hline $\begin{array}{c}\text { Dynamic } \\
\text { Range }\end{array}$ & $44 \mathrm{~dB}$ & $42 \mathrm{~dB}$ & $43 \mathrm{~dB}$ & $47 \mathrm{~dB}$ & $44 \mathrm{~dB}$ & $48 \mathrm{~dB}$ \\
\hline
\end{tabular}

Tab. 11 - Collected data relating to scans performed by a medical professional on Patient 4.

Tab. 12 - Collected data relating to scans performed by a medical professional on Patient 5 .

\begin{tabular}{|c|c|c|c|c|c|c|}
\cline { 2 - 7 } \multicolumn{1}{c|}{} & \multicolumn{2}{c|}{ Gluteus } & \multicolumn{2}{c|}{ Popliteal Fossa } & \multicolumn{2}{c|}{ Femoral Quadriceps } \\
\hline Average & sagittal & transverse & sagittal & transverse & sagittal & transverse \\
\hline Gain & $52 \%$ & $50 \%$ & $50 \%$ & $50 \%$ & $51 \%$ & $49 \%$ \\
\hline Depth & $5,1 \mathrm{~cm}$ & $5,2 \mathrm{~cm}$ & $5,1 \mathrm{~cm}$ & $5,3 \mathrm{~cm}$ & $4,8 \mathrm{~cm}$ & $4,6 \mathrm{~cm}$ \\
\hline Focus & $3,1 \mathrm{~cm}$ & $3 \mathrm{~cm}$ & $3,1 \mathrm{~cm}$ & $3 \mathrm{~cm}$ & $2,7 \mathrm{~cm}$ & $2,65 \mathrm{~cm}$ \\
\hline $\begin{array}{c}\text { Dynamic } \\
\text { Range }\end{array}$ & $49,2 \mathrm{~dB}$ & $48,2 \mathrm{~dB}$ & $48 \mathrm{~dB}$ & $49,4 \mathrm{~dB}$ & $49,4 \mathrm{~dB}$ & $49,8 \mathrm{~dB}$ \\
\hline
\end{tabular}

\begin{tabular}{|c|c|}
\hline Transducer 5-12 MHz & Preset on the US Equipment \\
\hline Gain & $46 \%$ \\
\hline Depth & $3 \mathrm{~cm}$ \\
\hline Focus & $1,5 \mathrm{~cm}$ \\
\hline Dynamic Range & $56 \mathrm{~dB}$ \\
\hline
\end{tabular}

Tab. 14 - Average of the collected data using the preset on the US equipment.

Tab. 13 - Average of the collected data relating to scans performed by a medical professional .

\begin{tabular}{|c|c|}
\hline Transducer 5-12 MHz & Optimized Preset \\
\hline Gain & $51 \%$ \\
\hline Depth & $4,5 \mathrm{~cm}$ \\
\hline Focus & $2,5 \mathrm{~cm}$ \\
\hline Dynamic Range & $47 \mathrm{~dB}$ \\
\hline
\end{tabular}

Tab. 15 - Average of the collected data using the optimized preset.

\begin{tabular}{|c|c|}
\hline Transducer 5- $12 \mathrm{MHz}$ & Medical Protocol \\
\hline Gain & $50 \%$ \\
\hline Depth & $5 \mathrm{~cm}$ \\
\hline Focus & $3 \mathrm{~cm}$ \\
\hline Dynamic Range & $49 \mathrm{~dB}$ \\
\hline
\end{tabular}

Tab. 16 - Average of the collected data relating to scans performed by a medical professional.

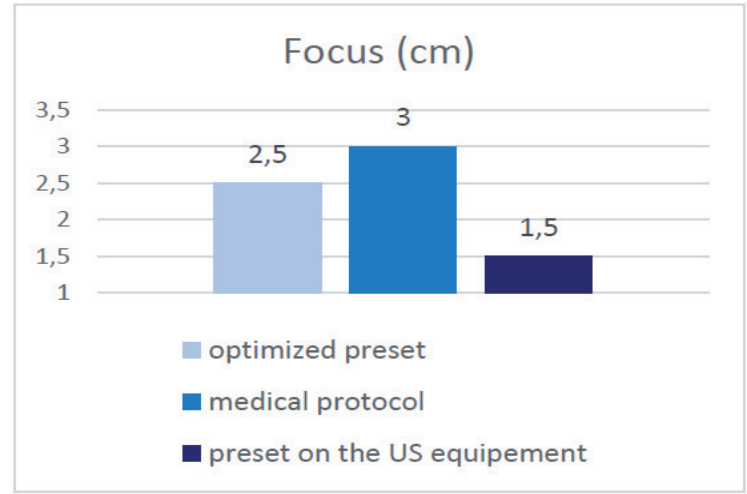

Tab. 17 - Gain (\%) comparison in the three protocols.

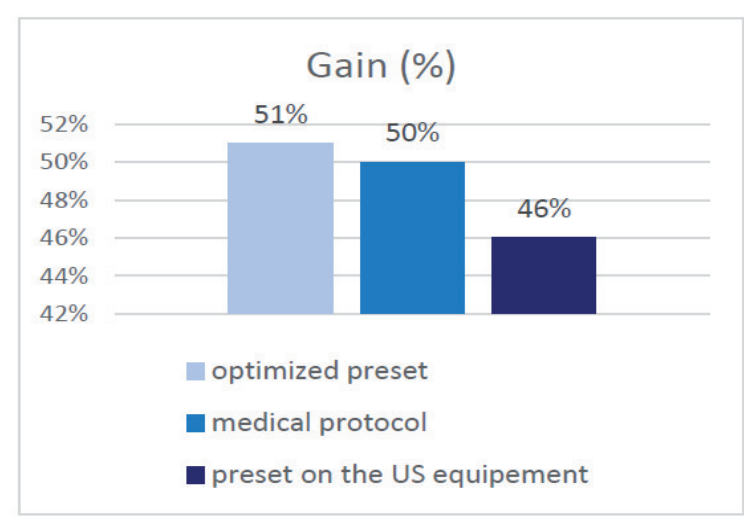

Tab. 19 - Focus $(\mathrm{cm})$ comparison in the three protocols.

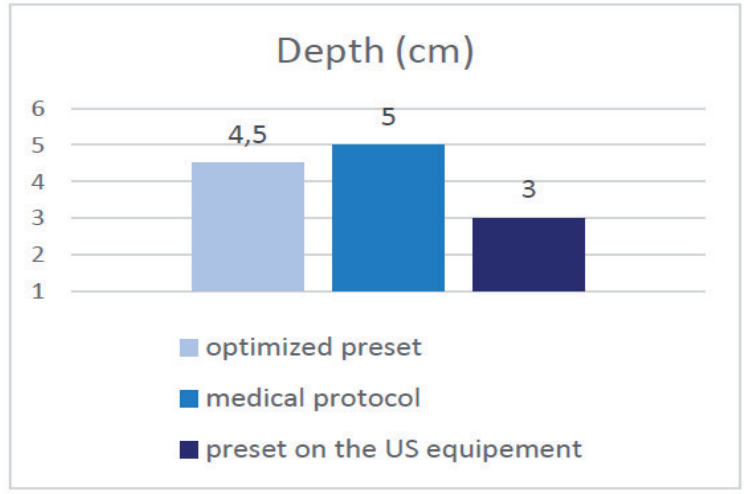

Talb. 18 - Depth $(\mathrm{cm})$ comparison in the three protocols.

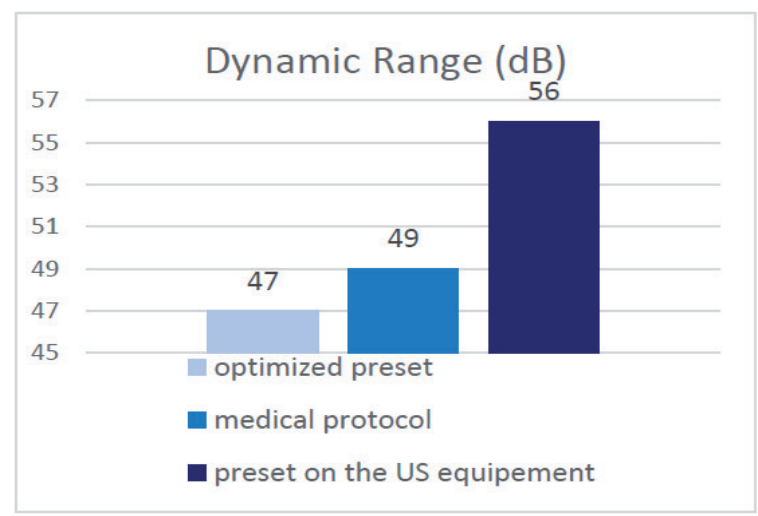

Tab. 20 - Dynamic range $(\mathrm{dB})$ comparison in the three protocols 


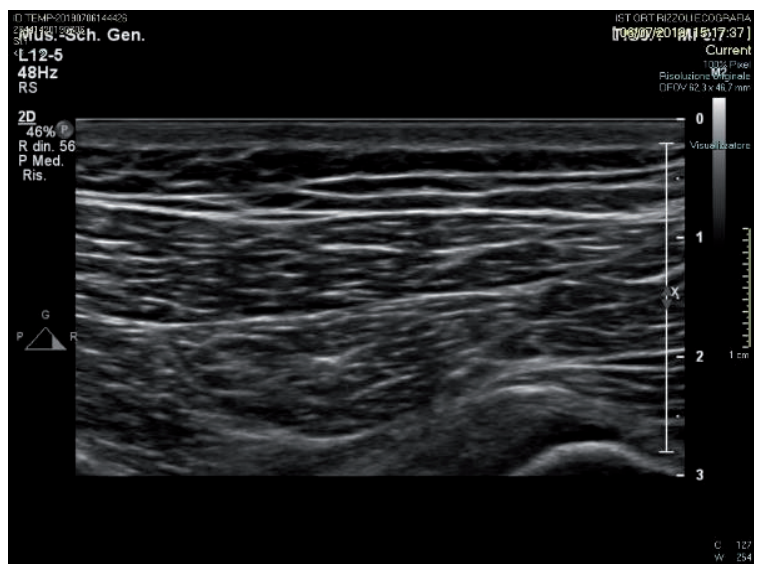

Fig. 6 - Sagittal ultrasound scan of the left gluteus performed by the use of the US equipement on patient 1 .

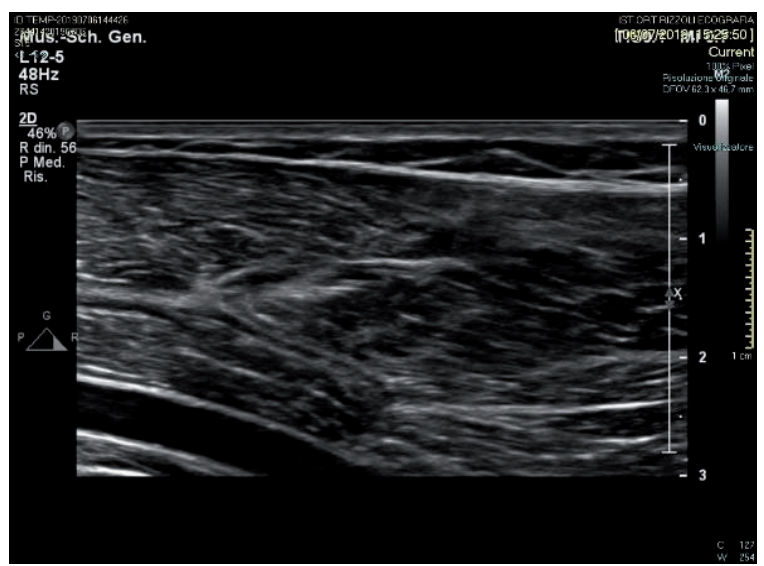

Fig. 8 - Sagittal ultrasound scan of the left popliteal fossa performed by the use of the US equipement on patient 1 .

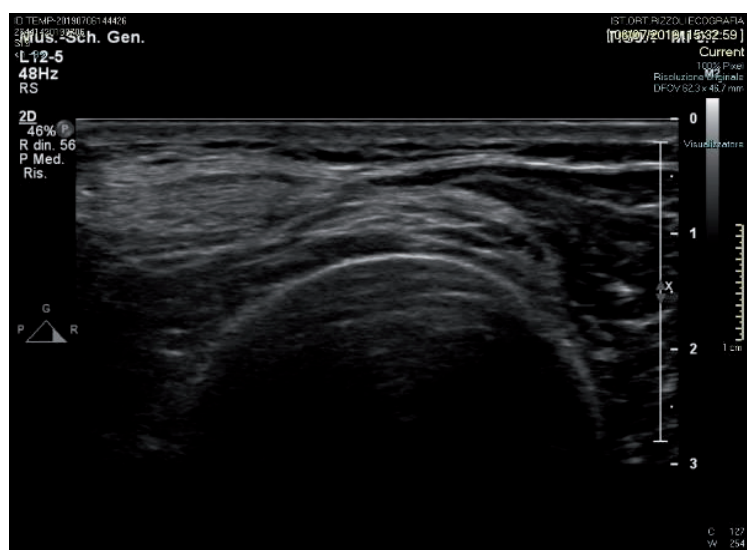

Fig. 10 - Sagittal ultrasound scan of the left femoral quadriceps performed by the use of the US equipement on patient 1.

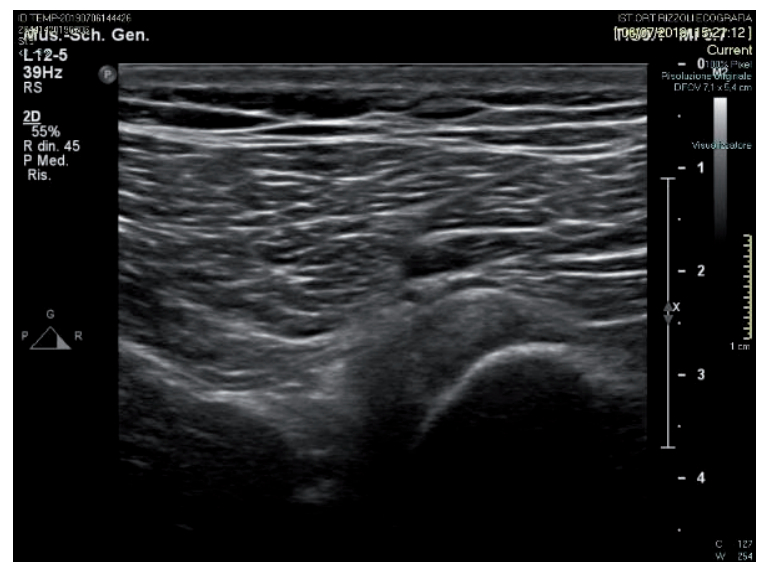

Fig. 7 - Sagittal ultrasound scan of the left gluteus performed by the use of the optimized Preset on patient 1.

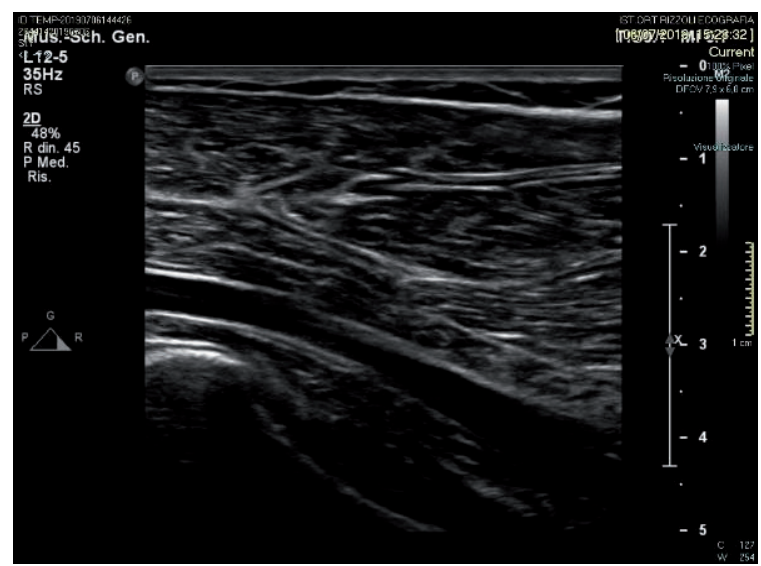

Fig. 9 - Sagittal ultrasound scan of the left popliteal fossa performed by the use of the optimized Preset on patient 1 .

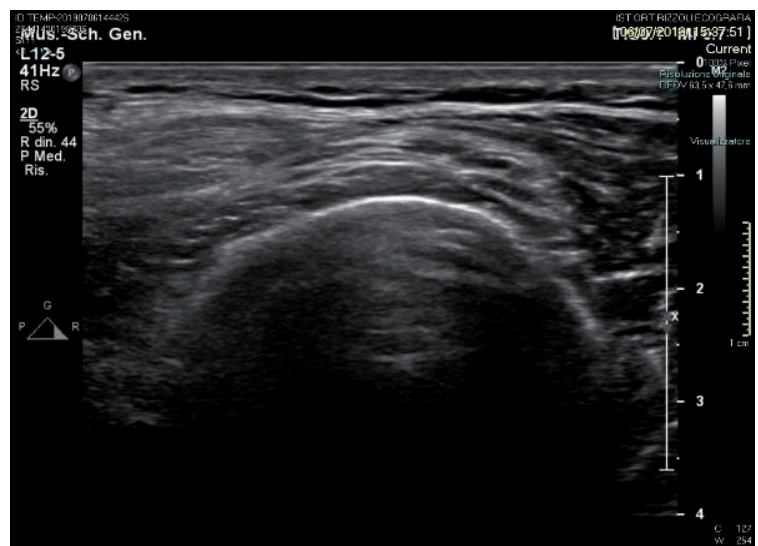

Fig. 11 - agittal ultrasound scan of the left femoral quadriceps performed by the use of the optimized Preset on patient 1.

\section{REFERENCES}

1. Porri Enrico, (November 2017) "Osservatorio parco installato: le apparecchiature di diagnostica per immagini in Italia", Centro Studi Assobiomedica, pp. 6-30.

2. American College of Emergency Physicians, (June 2016) "Ultrasound Guidelines: Emergency, Point-of-care, and Clinical Ultrasound Guidelines in Medicine", pp. 19-21; 24-27. [Online] Available: https://www.acep.org/globalassets/new-pdfs/policystatements/ultrasound-guidelines---emergency-point-of-care-and-clinical-ultrasound-guidelines-in-medicine.pdf.

3. K.Carmody, C.Moore e D.Feller-Kopman, (2012) "L'ecografia in medicina critica ed in emergenza", edited by M.Chiaranda, Padova, Piccin, pp. 1-6; 27-30

4. American Institute of Ultrasound in Medicine Bioeffects Committee, (2008) "Bioeffects Considerations for the Safety of Diagnostic Ultrasound", in Journal of Ultrasound in Medicine, 27:503-515, pp. 504-506.

5. M.Meola, (2008) "Principi fisici dell'ecografia, natura e caratteristiche fisiche degli ultrasuoni", in Ecografia clinica e color doppler in Nefrologia, Eureka, pp. 19-23.

6. A.Bonnin, J.P.Convard, P.Legmann, C.Broussouloux, (1996) "Ecografia", a cura di L. Di Guglielmo, Milano, Masson, pp. 4-5. 